\title{
STRUCTURAL MODEL OF SIMILARITY FOR FUZZY CLUSTERING
}

\author{
Mika Sato and Yoshiharu Sato*
}

\begin{abstract}
As a generalization of the additive clustering model (Shepard, R.N. and Arabie, P. (1979)), we discuss the following three additive fuzzy clustering models: a simple additive fuzzy clustering model, an overlapping fuzzy clustering model and a fuzzy clustering model for ordinal scaled similarity. The essential merits of fuzzy clustering models are 1) the amounts of computations for the identification of the models are much fewer than a hard clustering model and 2) a fewer number of clusters is needed to get a suitable fitness.

These fuzzy clustering models are extended to the model for asymmetric similarity. In this model, the concept of the similarity among clusters is introduced. The crucial assumption of this model is that the asymmetry of the similarity between the pair of objects is caused by the asymmetric similarity among clusters. The validity of this model is shown by some examples.
\end{abstract}

\section{Introduction}

The additive clustering model in hard cluster analysis is intended to find the structure of the similarity between the pair of objects. However, a large number of clusters is required by the constraint of the model. Usually, the model is denoted by the following:

$$
s_{i j} \approx \sum_{k=1}^{K} w_{k} p_{i k} p_{j k},
$$

where $s_{i j}(i, j=1,2, \cdots, n)$ is the similarity between objects $i$ and $j$, and $K$ is the number of clusters, $w_{k}$ is a weight representing the salience of the property corresponding to cluster $k$. If object $i$ has the property of cluster $k$ then $p_{i k}=1$, otherwise is 0 . Notice that the product $p_{i k} p_{j k}$ is unity if and only if both objects $i$ and $j$ belong to cluster $k$ and the similarity between the pair of objects is defined to be the common property of objects. Moreover, if the pair of objects shares some common properties, the grade which the pair of objects contribute to the similarities is assumed to be mutually independent (Shepard, R.N. and Arabie, P. (1979)).

On the other hand, in the case of the fuzzy clustering, a fuzzy cluster is defined to be a fuzzy subset on the set of objects and a fuzzy grade of each object represents the degree of belongingness. If we assume that a cluster is the group whose elements share common properties, then the fuzzy grade shows the degree of which the object has the common

*Division of Information Engineering, Hokkaido University

Key words: Similarity data; Structural analysis; Fuzzy cluster; Asymmetric similarity; Additive clustering model. 
properties of each cluster. By introducing the concept of the fuzzy cluster into the additive clustering model, we can construct a natural clustering model which it is possible to interpret as having the structure of similarity by using fewer clusters.

In this paper, we propose the simple additive fuzzy clustering model, the overlapping additive fuzzy clustering model, and the additive fuzzy clustering model for ordinal scaled similarity. Furthermore, we discuss the case that the observed similarity data is asymmetric, and extend the model to asymmetric similarity.

\section{Additive fuzzy clustering model for symmetric similarity}

\subsection{Simple additive fuzzy clustering model}

We define the simple additive fuzzy clustering model as follows:

$$
s_{i j} \approx \alpha \sum_{k=1}^{K} u_{i k} u_{j k},
$$

where $\alpha$ is non-negative constant, the similarity $s_{i j}$ has a ratio scale and $0 \leq s_{i j} \leq 1 . u_{i k}$ is a fuzzy grade which represents the degree of belongingness of object $i$ to cluster $k$. Generally, $u_{i k}$ are denoted by using the matrix representation $U=\left(u_{i k}\right)$ called a partition matrix, which satisfy the following condition:

$$
u_{i k} \geq 0, \quad \sum_{k=1}^{K} u_{i k}=1 .
$$

In this case, the product $u_{i k} u_{j k}$ is the degree of simultaneous belongingness of objects $i$ and $j$ to cluster $k$. That is, the product denotes the degree of the sharing of common properties. And we introduce the positive constant $\alpha$ to control the goodness of fit between $s_{i j}$ and $\sum_{k=1}^{K} u_{i k} u_{j k}$, because the constraint of $u_{i k} u_{j k}$ exists (by (2.2)). Namely, we assume that $s_{i j}$ is proportional to $\sum_{k=1}^{K} u_{i k} u_{j k}$. Moreover, in the case that $s_{i j}$ has an interval scale, we define the following model:

$$
s_{i j} \approx \alpha \sum_{k=1}^{K} u_{i k} u_{j k}+\beta,
$$

where $\beta$ is an additive constant. If the similarity $s_{i j}$ has an interval scale, then the origin of $s_{i j}$ is arbitrary, and $s_{i j}$ need not satisfy $s_{i j} \geq 0$. Assuming that $\zeta_{i j}$ has an absolute scale which determines the unit and the origin uniquely, and $s_{i j}$ is observed in a certain unit, then we may adjust the unit by the following:

$$
s_{i j}=\alpha \zeta_{i j} \quad(\alpha>0)
$$

And if $s_{i j}$ has an interval scale, then it is represented by the following:

$$
s_{i j}=\alpha^{\prime} \zeta_{i j}+\beta\left(\alpha^{\prime}>0, \beta \neq 0\right) .
$$

Consequently, we propose the model (2.3).

The method of the fuzzy clustering based on this model is to find the partition matrix $U=\left(u_{i k}\right)$ which satisfies the condition (2.2) and has the best fitness for the model (2.1) 
or (2.3). Then we find $U$ and $\alpha$ which minimize the following normalized sum of squared errors, $\eta_{1}^{2}$, under the condition (2.2),

$$
\eta_{1}^{2}=\frac{\sum_{i \neq j=1}^{n}\left(s_{i j}-\alpha \sum_{k=1}^{K} u_{i k} u_{j k}\right)^{2}}{\sum_{i \neq j=1}^{n}\left(s_{i j}-\bar{s}\right)^{2}}, \quad \bar{s}=\frac{1}{n(n-1)} \sum_{i \neq j=1}^{n} s_{i j} .
$$

In this case, we introduce the following transformation, to avoid the constrained optimization problem:

$$
\left(\begin{array}{c}
u_{i 1} \\
u_{i 2} \\
u_{i 3} \\
\vdots \\
u_{i K-1} \\
u_{i K}
\end{array}\right)=\left(\begin{array}{l}
\cos ^{2} \theta_{i 1} \\
\sin ^{2} \theta_{i 1} \cos ^{2} \theta_{i 2} \\
\sin ^{2} \theta_{i 1} \sin ^{2} \theta_{i 2} \cos ^{2} \theta_{i 3} \\
\vdots \\
\sin ^{2} \theta_{i 1} \sin ^{2} \theta_{i 2} \cdots \cos ^{2} \theta_{i K-1} \\
\sin ^{2} \theta_{i 1} \sin ^{2} \theta_{i 2} \cdots \sin ^{2} \theta_{i K-1}
\end{array}\right)
$$

From this, we know that the condition (2.2) is satisfied for any $\theta_{i k} \in[0,2 \pi]$, that is,

$$
\begin{gathered}
\sum_{k=1}^{K} u_{i k}\left(\theta_{i 1}, \theta_{i 2}, \cdots, \theta_{i K-1}\right)=1, \\
u_{i k}\left(\theta_{i 1}, \theta_{i 2}, \cdots, \theta_{i K-1}\right) \geq 0 .
\end{gathered}
$$

\subsection{Overlapping additive fuzzy clustering model}

This model is defined by the following:

$$
s_{i j} \approx \tilde{\alpha} \sum_{k=1}^{K} u_{i k} u_{j k} .
$$

We loosen the constraint of condition (2.2) and assume

$$
0 \leq u_{i k} \leq 1
$$

The looseness of condition (2.2) makes us consider the concept of overlapping flexibly. That is, it allows that objects belong to all clusters or no clusters. In this case, the condition $0<$ $\tilde{\alpha} \leq 1$ is needed, because when $\tilde{\alpha} \sum_{k=1}^{K} u_{i k} u_{j k}=$ const., if $\tilde{\alpha} \rightarrow \infty$ then $\sum_{k=1}^{K} u_{i k} u_{j k} \rightarrow 0$. In order to avoid the constrained problem, we use the following transformations:

$$
\begin{aligned}
& \tilde{\alpha}=\frac{1}{1+e^{-a}}, \\
& u_{i k}=\sin ^{2} \theta_{i k}
\end{aligned}
$$

where $-\infty<a<\infty, \theta_{i k} \in\left[0, \frac{\pi}{2}\right]$, and notice that $\tilde{\alpha}$ does not attain to 1 , but it attains to 1 approximately. 


\subsection{Additive fuzzy clustering model for ordinal similarity}

The model is defined as follows:

$$
s_{i j} \stackrel{m}{=} \sum_{k=1}^{K} u_{i k} u_{j k},
$$

where $\stackrel{m}{=}$ denotes a monotone relation. If we denote $w_{i j}=\sum_{k=1}^{K} u_{i k} u_{j k}$, then we assume the following relation:

$$
\left\{\begin{array}{c}
s_{i j}<s_{h l} \Rightarrow w_{i j} \leq w_{h l} \\
s_{i j}=s_{h l} \Rightarrow w_{i j}=w_{h l} \\
i, j, h, l=1, \cdots, n .
\end{array}\right.
$$

To simplify this, we rewrite $W=f(U)$ and $S=\left(s_{i j}\right)$, then (2.9) is replaced by

$$
S \stackrel{m}{=} W=f(U) \text {. }
$$

The purpose is to find such a solution $U$, using this model (2.9), satisfying a monotone relation between $S$ and $W$. That is, when we introduce $\tilde{W}=T(S)$, where $T$ denotes a monotone transformation, we may fit $\tilde{W}$ to $W=f(U)$ by using the least squares method (Kruskal, J.B. (1964), Kruskal, J.B. (1964)). This process is represented by

$$
S \stackrel{m}{=} \tilde{W} \cong W=f(U),
$$

where $\cong$ means an approximation solved by the least squares method. The practical calculation is to determine $\tilde{W}$ by using $S$ and $W$ based on the monotone regression principle (Kruskal, J.B. (1964)), and to find $W$, which minimizes the following $\eta_{2}^{2}$ :

$$
\eta_{2}^{2}=\frac{\sum_{i \neq j=1}^{n}\left(w_{i j}-\tilde{w}_{i j}\right)^{2}}{\sum_{i \neq j=1}^{n}\left(w_{i j}-\bar{w}\right)^{2}}, \quad \bar{w}=\frac{1}{n(n-1)} \sum_{i \neq j=1}^{n} w_{i j},
$$

under the condition $(2.2)$.

\section{Reproduction and robustness}

In a model fitting problem, it is important to see whether the algorithm of the fitting is able to reproduce the structure or not when the observed data actually have the structure of the model. And whether the small change of the data has an extreme influence on the result or not is also an important problem. The former is known as a reproduction property and the latter is a robustness of the model. By the Monte Carlo simulation, we shall investigate these problems. At first, we show the outline of the simulation for the reproduction.

(step 1) Generate the matrix $\Theta=\left(\theta_{i k}\right)$ using uniform pseudorandom numbers in the interval $\left[0, \frac{\pi}{2}\right]$. Calculate $U=U(\Theta)$ and $S=U(\Theta) U(\Theta)^{t}$, where $t$ denotes a transpose.

(step 2) Regarding $S$ as given similarity data, estimate $U^{*}$ of each fuzzy clustering model using the initial matrix $U^{(0)}=U(\Theta+\Delta \Theta)$, where the matrix $\Delta \Theta$ is generated by uniform pseudorandom numbers in the interval $\left[0, \frac{\pi}{2}\right]$. 
(step 3) Calculate the sum of squared errors

$$
\eta_{u}^{2} \equiv \sum_{i=1}^{n} \sum_{k=1}^{(K)}\left(u_{i k}-u_{i k}^{*}\right)^{2},
$$

where $u_{i k}$ and $u_{i k}^{*}$ are elements of matrices $U$ and $U^{*}$, respectively, and $(K)$ denotes $K-1$ in the case of the simple additive clustering model, and equals $K$ in the case of the overlapping additive clustering model.

And in the case of a fuzzy additive clustering model for rank ordered similarity, it seems to be reasonable that the error from the true structure is measured by a rank correlation, because the clustering is based on the information of the rank orders of similarity. Then we use the following Spearman's rank correlation coefficient. If we assume that $T_{l}, T_{l}^{*}(l=1, \cdots, N=$ $\left.\frac{n(n-1)}{2}\right)$ are the rank orders of $\tau_{i j} \equiv \sum_{k=1}^{K} u_{i k} u_{j k}, \tau_{i j}^{*} \equiv \sum_{k=1}^{K} u_{i k}^{*} u_{j k}^{*}$, respectively, then the rank correlation between $T_{l}$ and $T_{l}^{*}$ is

$$
\rho_{T}=1-\frac{6 \sum_{l=1}^{N}\left(T_{l}-T_{l}^{*}\right)^{2}}{N\left(N^{2}-1\right)} .
$$

In the case of a rank ordered additive clustering, we calculate $\rho_{T}$ at step 3.

The above simulation is repeated 50 times at each situation, the numbers of observations are 10, 20 and 30. The mean and the standard deviation of $\eta_{u}^{2}$ (or $\rho_{T}$ ) are calculated. The results are Tables 3.1, 3.2, 3.3. These tables show that these algorithms have a reproduction property, because the mean and the standard deviation of the sum of the squared errors are approximately 0 in the case of the simple additive clustering model or the overlapping additive clustering model. And in the case of the model for rank ordered similarity, the structure of the rank order is completely reproduced, because the data do not include observed errors.

Moreover, the way of simulation for the robustness is shown as follows:

(step 1) Using uniform pseudorandom numbers in the interval $\left[0, \frac{\pi}{2}\right]$, the matrix $\Theta=$ $\left(\theta_{i k}\right),(i=1,2, \cdots, n, k=1,2, \cdots,(K))$ is generated. Calculate $U=U(\Theta)$ and $S=U(\Theta) U(\Theta)^{t}$, where $t$ denotes a transpose.

(step 2) Generate the error $\Delta S$ using the pseudorandom numbers, and calculate the following:

$$
S^{*}=S+\Delta S
$$

(step 3) Regarding $S^{*}$ as given similarity data, estimate $U^{*}$ of each fuzzy clustering model using the initial matrix $U^{(0)}=U(\Theta+\Delta \Theta)$, where the matrix $\Delta \Theta$ is generated by the uniform pseudorandom numbers in the interval $\left[0, \frac{\pi}{2}\right]$.

(step 4) Using $S, S^{*}$ and $U, U^{*}$, calculate,

$$
\epsilon_{s}^{2} \equiv \frac{\sum_{i \neq j=1}^{n}\left(s_{i j}-s_{i j}^{*}\right)^{2}}{\sum_{i \neq j=1}^{n}\left(s_{i j}-\bar{s}\right)^{2}}, \quad \bar{s}=\frac{1}{n(n-1)} \sum_{i \neq j=1}^{n} s_{i j} .
$$




$$
\epsilon_{u}^{2} \equiv \frac{\sum_{i=1}^{n} \sum_{k=1}^{(K)}\left(u_{i k}-u_{i k}^{*}\right)^{2}}{\sum_{i=1}^{n} \sum_{k=1}^{(K)}\left(u_{i j}-\bar{u}\right)^{2}}, \quad \bar{u}=\frac{1}{n(K)} \sum_{i=1}^{n} \sum_{k=1}^{(K)} u_{i k} .
$$

$(K)$ denotes $K-1$ in the case of the simple additive clustering model, and equals $K$ in the case of the overlapping additive clustering model.

The above simulation is repeated 60 times as the number of observations $n=15$. The result is shown by Figure 3.1-Figure 3.4. In these figures, the abscissa shows the normalized error of similarity (3.1) and the ordinate shows the normalized error of grade (3.2), and the value of $K$ is the number of clusters. From the results of these simulations, we can find the robustness of the models, because when the similarity has moderate error, the errors of result are smaller than the error of the similarity data. Furthermore, we examine the robustness in the case of the model for rank ordered similarity. We arrange $s_{i j}, s_{i j}^{*}, \tau_{i j} \equiv \sum_{k=1}^{K} u_{i k} u_{j k}$, $\tau_{i j}^{*} \equiv \sum_{k=1}^{K} u_{i k}^{*} u_{j k}^{*}$ according to the rankings. Their rank orders represented by $R_{l}, R_{l}^{*}, T_{l}$ and $T_{l}^{*}\left(l=1, \cdots, N=\frac{n(n-1)}{2}\right)$, then the rank correlation coefficients are the following:

$$
\begin{gathered}
\rho_{R}=1-\frac{6 \sum_{l=1}^{N}\left(R_{l}-R_{l}^{*}\right)^{2}}{N\left(N^{2}-1\right)} \\
\rho_{T}=1-\frac{6 \sum_{l=1}^{N}\left(T_{l}-T_{l}^{*}\right)^{2}}{N\left(N^{2}-1\right)} .
\end{gathered}
$$

In the above simulation, if we replace the expressions (3.1) and (3.2) with $1-\rho_{R}, 1-\rho_{T}$, then we get Figures 3.5 and 3.6 which show the results of 60 times where the number of objects is 15 . In this figure, the abscissa is $1-\rho_{R}$, and the ordinate is $1-\rho_{T}$. In this simulation, we can find the robustness, because the results are not so far from the real structure when the errors are moderate. 
Table 3.1 Reproduction of structure of clusters

(Simple additive fuzzy clustering model)

\begin{tabular}{|c|c|c|c|}
\hline \multirow{2}{*}{ number of objects } & \multirow{2}{*}{$\begin{array}{c}\text { the sum of } \\
\text { the square error }\end{array}$} & \multicolumn{2}{|c|}{ number of clusters } \\
\cline { 3 - 4 } & mean & $.46 \times 10^{-05}$ & $.39 \times 10^{-04}$ \\
\hline \hline \multirow{2}{*}{10} & standard deviation & $.67 \times 10^{-05}$ & $.54 \times 10^{-04}$ \\
\hline \multirow{2}{*}{20} & mean & $.12 \times 10^{-05}$ & $.93 \times 10^{-05}$ \\
\cline { 2 - 4 } & standard deviation & $.76 \times 10^{-06}$ & $.98 \times 10^{-05}$ \\
\hline \hline \multirow{2}{*}{30} & mean & $.49 \times 10^{-06}$ & $.59 \times 10^{-05}$ \\
\cline { 2 - 4 } & standard deviation & $.31 \times 10^{-06}$ & $.38 \times 10^{-05}$ \\
\hline
\end{tabular}

Table 3.2 Reproduction of structure of clusters

(Overlapping additive fuzzy clustering model)

\begin{tabular}{|c|c|c|c|}
\hline \multirow{2}{*}{ number of objects } & \multirow{2}{*}{$\begin{array}{c}\text { the sum of } \\
\text { the square error }\end{array}$} & \multicolumn{2}{|c|}{ number of clusters } \\
\cline { 3 - 4 } & mean & $.93 \times 10^{-04}$ & $.25 \times 10^{-04}$ \\
\hline \hline \multirow{2}{*}{10} & standard deviation & $.42 \times 10^{-03}$ & $.16 \times 10^{-04}$ \\
\hline \hline \multirow{2}{*}{20} & mean & $.72 \times 10^{-05}$ & $.92 \times 10^{-05}$ \\
\cline { 2 - 4 } & standard deviation & $.68 \times 10^{-05}$ & $.51 \times 10^{-05}$ \\
\hline \hline \multirow{2}{*}{30} & mean & $.40 \times 10^{-05}$ & $.44 \times 10^{-05}$ \\
\cline { 2 - 4 } & standard deviation & $.25 \times 10^{-05}$ & $.18 \times 10^{-05}$ \\
\hline
\end{tabular}

Table 3.3 Reproduction of structure of clusters

(Additive fuzzy clustering model for ordinal similarity)

\begin{tabular}{|c|c|c|c|}
\hline \multirow{2}{*}{ number of objects } & \multirow{2}{*}{$\begin{array}{c}\text { order } \\
\text { correlation coefficient }\end{array}$} & \multicolumn{2}{|c|}{ number of clusters } \\
\cline { 3 - 4 } & mean & 1.0 & 3 \\
\hline \hline \multirow{2}{*}{10} & standard deviation & 0.0 & 1.0 \\
\cline { 2 - 4 } & mean & 1.0 & 0.0 \\
\hline \hline \multirow{2}{*}{20} & standard deviation & 0.0 & 0.0 \\
\cline { 2 - 4 } & mean & 1.0 & 1.0 \\
\hline \hline \multirow{2}{*}{30} & standard deviation & 0.0 & 0.0 \\
\cline { 2 - 4 } & & & \\
\end{tabular}




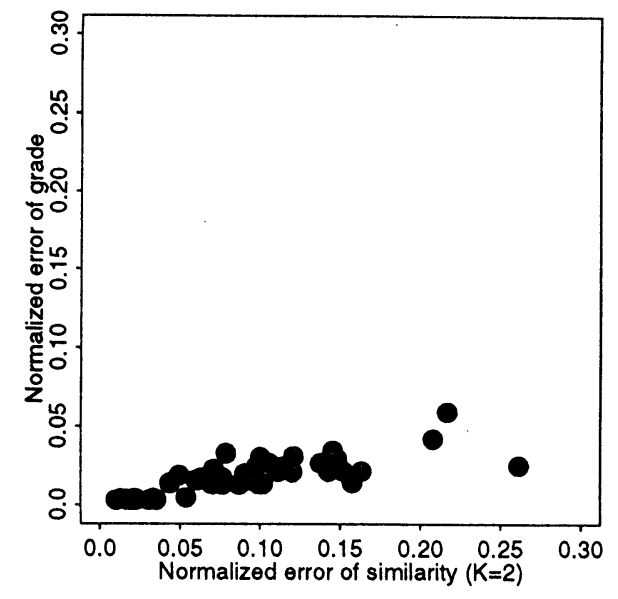

Figure 3.1 Robustness of simple model

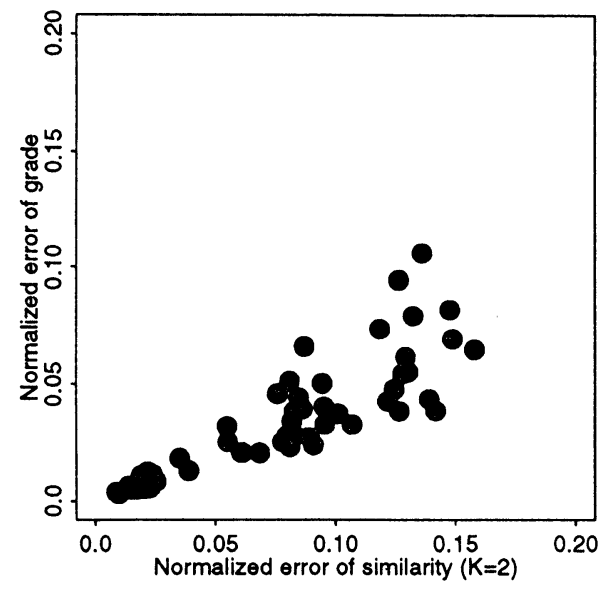

Figure 3.3 Robustness of overlapping model

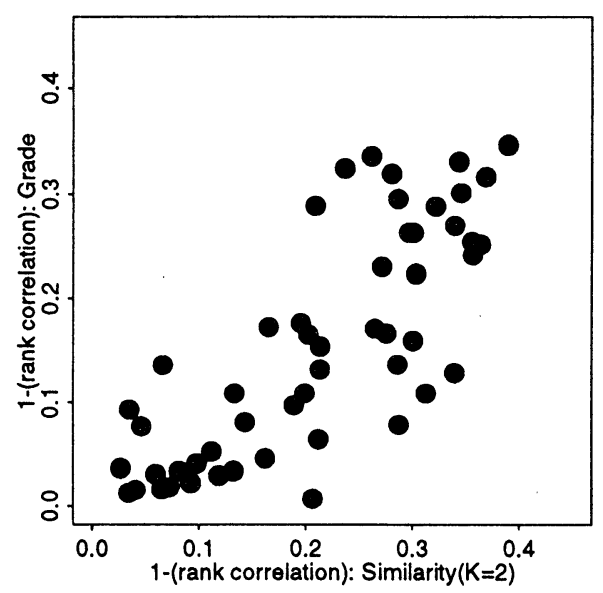

Figure 3.5 Robustness of ordered model

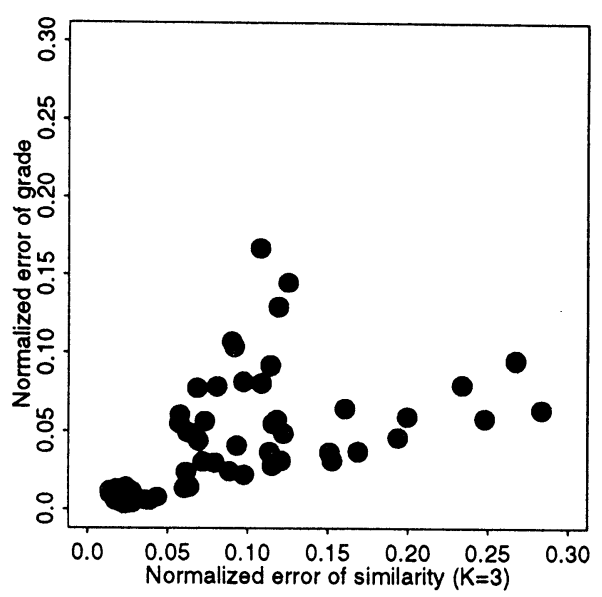

Figure 3.2 Robustness of simple model

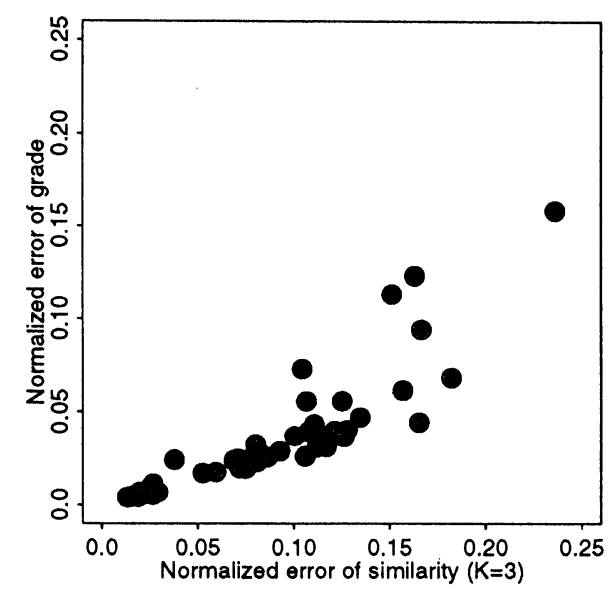

Figure 3.4 Robustness of overlapping model

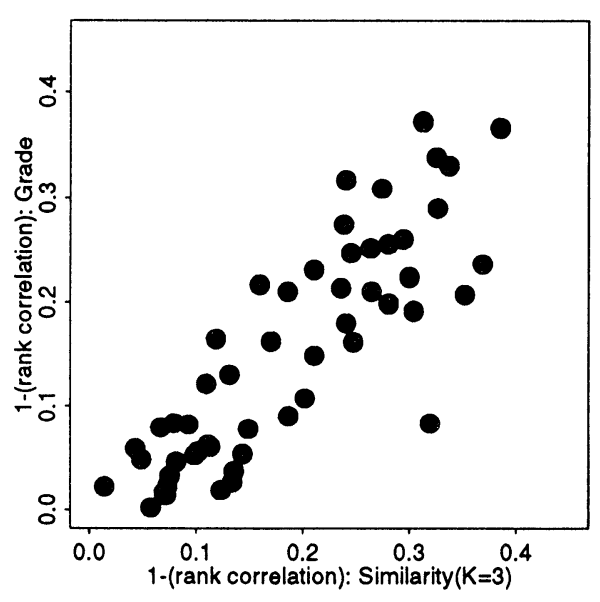

Figure 3.6 Robustness of ordered model 


\section{Uniqueness of solutions}

In the simple additive fuzzy clustering model, the expressions (2.1) and (2.2) are denoted as follows:

$$
S \approx \alpha U U^{t}
$$

where $U \mathbf{1}_{K}=\mathbf{1}_{n}, \quad U \geq 0$ (all elements are non-negative), and $\mathbf{1}_{l}$ shows an $l$-dimensional vector in which all elements are 1 , and $t$ denotes a transpose. $T$ is assumed to be an arbitrary $K$ dimensional orthogonal matrix, and $V$ is assumed to be an $(n \times K)$-matrix which satisfies $U=V T$. Then we get

$$
\begin{aligned}
S \approx \alpha U U^{t} & =\alpha(V T)(V T)^{t} \\
& =\alpha V T T^{t} V^{t} \\
& =\alpha V V^{t} .
\end{aligned}
$$

However, the condition $V 1_{K}=1_{n}, V \geq 0$ are not always satisfied. That is, generally, $V$ is not the grade of fuzzy clusters. Then we find the region of $U$ when $V$ is the grade of fuzzy clusters by an arbitrary orthogonal rotation, namely the condition

$$
V \mathbf{1}_{K}=\mathbf{1}_{n}, \quad V \geq 0
$$

is satisfied. Now $\left(u_{i 1}, u_{i 2}, \cdots, u_{i K}\right)$ are assumed to be grades of fuzzy clusters with respect to $i$-th object, and this is considered to be a coordinate of $i$-th object $u_{i}=\left(u_{i 1}, u_{i 2}, \cdots, u_{i K}\right)(i=$ $1,2, \cdots, n)$ with respect to an arbitrary $K$ dimensional orthogonal base $\left\{e_{1}, e_{2}, \cdots, e_{K}\right\}$, namely

$$
\begin{aligned}
u_{i} & =\left(u_{i 1}, u_{i 2}, \cdots, u_{i K}\right) \\
& =u_{i 1} e_{1}+u_{i 2} e_{2}+\cdots+u_{i K} e_{K} .
\end{aligned}
$$

From the condition $u_{i k} \geq 0, \quad \sum_{k=1}^{K} u_{i k}=1, \mathbf{u}_{i}$ is a convex combination of $e_{1}, e_{2}, \cdots, e_{K}$. Since $e_{1}, e_{2}, \cdots, e_{K}$ is linearly independent, $\mathbf{u}_{i}$ is contained in $(K-1)$-simplex. On the hyperplane $Z: z_{1}+z_{2}+\cdots+z_{K}=1$, suppose that $T^{t}$ is any rotation around the normal vector of $Z$, then for any $\mathrm{u} \in Z, \mathbf{v}=T^{t} \mathbf{u}$ is contained in $Z$. Therefore, if $\mathbf{u}_{i}$ is contained in the inscribed hypersphere of the $(K-1)$-simplex, then the solution is not unique with respect to rotation $T^{t}$ around the normal vector of $(K-1)$-simplex. Generally, the proportion of the volume of the $(K-1)$-simplex $V S I$ to the volume of the inscribed hypersphere $V S$ is

$$
f_{K}=\frac{V S}{V S I}=\frac{(K-1) ! \pi^{\frac{K-1}{2}}}{\Gamma\left(\frac{K-1}{2}+1\right) \sqrt{K}(K(K-1))^{\frac{K-1}{2}}}
$$

In each of the cases that the number of clusters is $3,4,5$, the proportion of the region of indefinite solution to the region of the feasible solution is the following:

Table 4.1 Proportion of the region

\begin{tabular}{|c|c|c|c|}
\hline$K$ & 3 & 4 & 5 \\
\hline$f_{K}$ & 0.60 & 0.30 & 0.66 \\
\hline
\end{tabular}

For all objects, the proportion is $\left(f_{K}\right)^{n}$. Then the solution is almost unique. 


\section{Additive fuzzy clustering model for asymmetric similarity}

\subsection{Simple additive fuzzy clustering model for asymmetric similarity}

If the observed similarity data is asymmetric, then the proposed additive fuzzy clustering models in the foregoing sections are not available. Then we extend the model (2.1) as follows:

$$
\tilde{s}_{i j} \approx \sum_{k=1}^{K} \sum_{l=1}^{K} w_{k l} u_{i k} u_{j l},
$$

where the observed asymmetric similarity $\tilde{s}_{i j}$ has a ratio scale and $0 \leq \tilde{s}_{i j} \leq 1 . u_{i k}$ is a fuzzy grade (the degree of belonging) in which object $i$ belongs to cluster $k$. The condition (2.2) is also assumed:

$$
u_{i k} \geq 0, \sum_{k=1}^{K} u_{i k}=1 .
$$

In this model, the weight $w_{k l}$ is considered to be a quantity which shows the asymmetric similarity between the pair of clusters. That is, we assume that the asymmetry of the similarity between the objects is caused by the asymmetry of the similarity between the clusters. If $k=l, u_{i k}, u_{j l}=1$ and $w_{k l}>1$, then the right hand side of (5.1) clearly exceed 1.0. Hence we need at least the following condition

$$
0 \leq w_{k l} \leq 1
$$

because $0 \leq \tilde{s}_{i j} \leq 1$. If the model (5.1) has the following condition

$$
w_{k l}=0(k \neq l), \quad \forall k \quad w_{k k}=\alpha,
$$

where $\alpha=$ const., then the model (5.1) identifies the model (2.1). That is, the model (2.1) is the special case of model (5.1).

We avoid the constrained problem by the transformation (2.5) and

$$
w_{k l}=\frac{1}{1+e^{-b}}
$$

where $-\infty<b<\infty$, and notice that $w_{k l}$ does not attain to 0 or 1 , but it attains to them approximately.

5.2. Overlapping additive fuzzy clustering model for asymmetric similarity

The model is defined by the following:

$$
\tilde{s}_{i j} \approx \sum_{k=1}^{K} \sum_{l=1}^{K} w_{k l} u_{i k} u_{j k} .
$$

This model is an extended model of (2.6). In this model, we also assume that

$$
0 \leq u_{i k} \leq 1, \quad 0 \leq w_{k l} \leq 1
$$

And we use the transformations (2.8) and (5.2) for the optimization process. 


\section{Numerical example}

We shall show the application of the fuzzy clustering model for the dissimilarity data among the terms of a kinship (Rosenberg, S. and Kim, M.P. (1975)) (Table 6.1). This dissimilarity is transformed to a similarity as follows:

$$
s_{i j}=1-\frac{d_{i j}}{d_{\max }}, \quad d_{\max } \equiv \max _{i, j} d_{i j} .
$$

Table 6.2 shows the matrix of the transformed similarity.

Table 6.1 Dissimilarity between terms of kinship

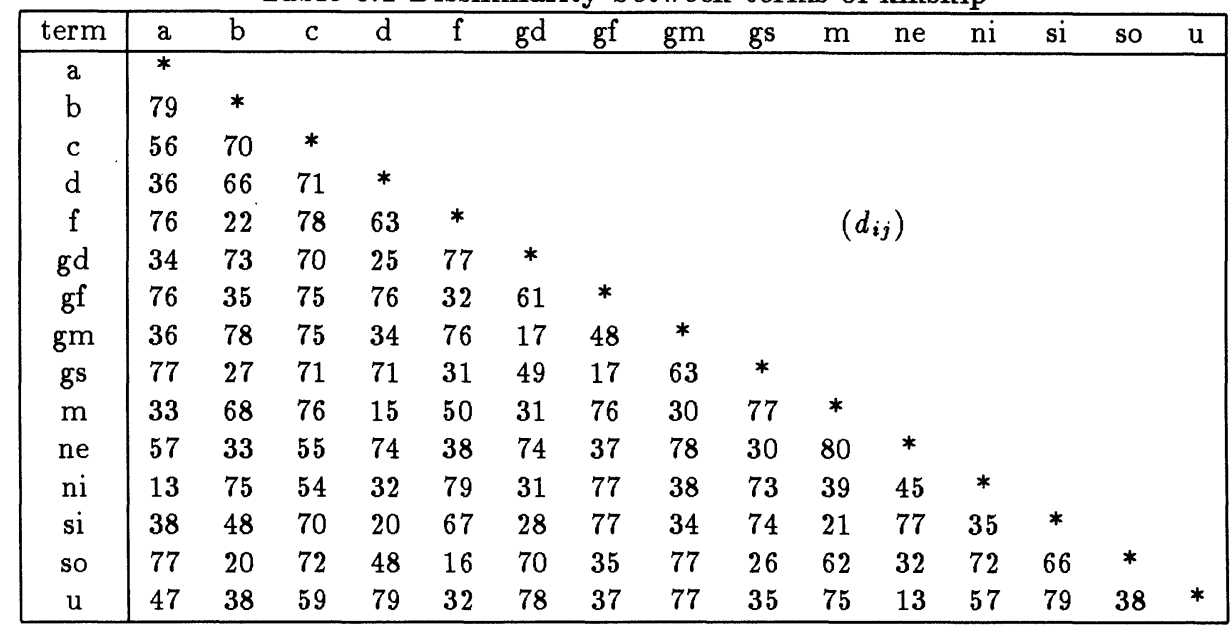

Table 6.2 Similarity between terms of kinship

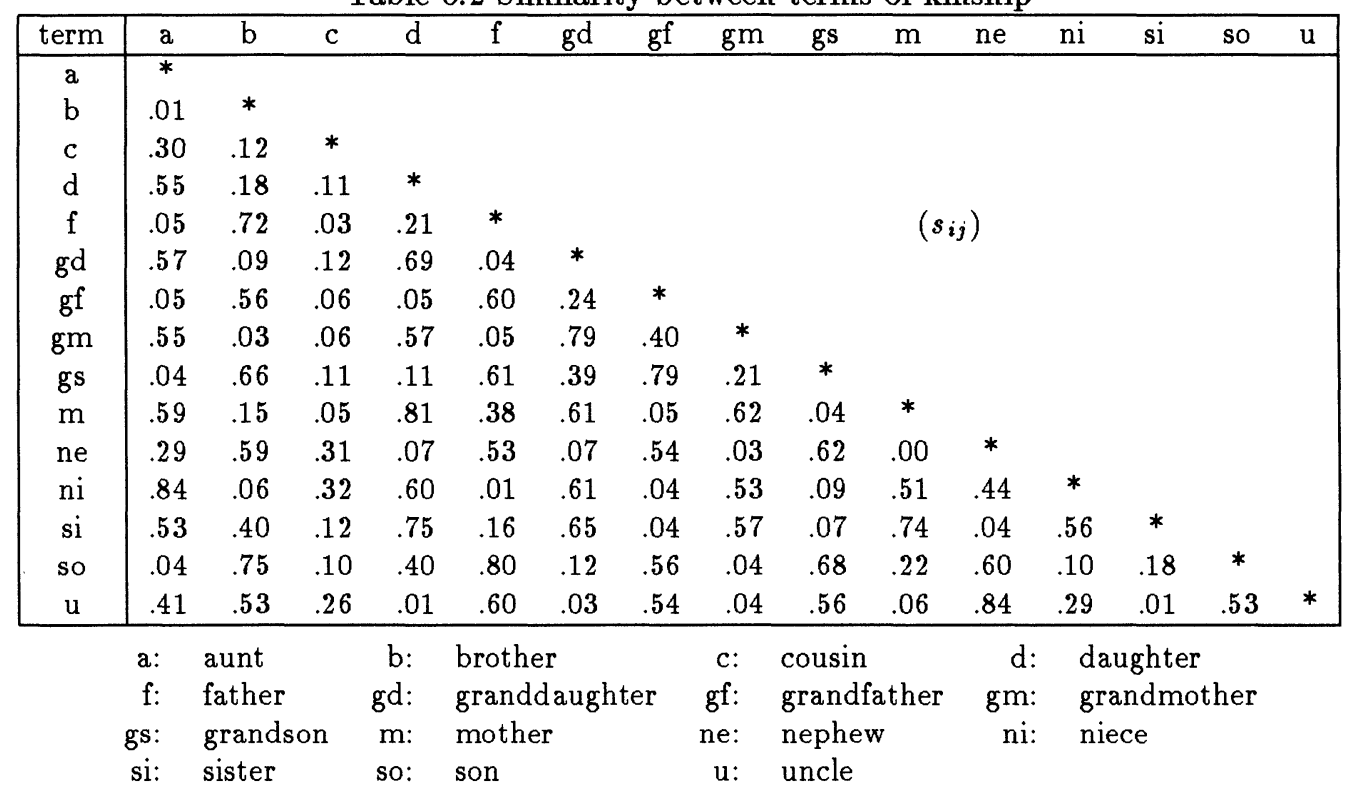


The optimization algorithm used in this example is shown in the appendix. In the algorithm, 20 sets of initial values are given by using uniform pseudorandom numbers in the interval $\left[0, \frac{\pi}{2}\right]$, and finally we select the best result. The number of clusters is determined based on the value of fitness. Figure 6.1 and Figure 6.2 show the variation of the value of fitness with respect to the number of clusters.

Increasing the number of clusters, the value of fitness decreases, but even if the number of clusters is greater than 3 , the decrease of the fitness is not so much. From the principle of parsimony, it should be considered that the number of clusters is determined to be 3 .

The results of the analysis are shown in Tables $6.3,6.4$ and 6.5. Table 6.3 is the result of the simple additive fuzzy clustering method, Table 6.4 is the result of the overlapping additive fuzzy clustering, and Table 6.5 shows the result of the rank ordered information of Table 6.2. $C_{1}, C_{2}, C_{3}$ represent three clusters, and each value denotes the fuzzy grade that a term belongs to each cluster. And $\eta_{1}^{2}, \eta_{1}^{\prime 2}, \eta_{2}^{2}$ show the value of fitness for each method. Figures 6.3,6.4 and 6.5 are visualizations of these results. In the abscissa, the terms of the kinship are located, and the ordinate shows the degree of belonging of clusters. Each part of the drawing represents the degree of belonging for each cluster. From this, the large part represented by using an oblique line upward to the right means female. And the terms whose degree of belonging is represented by the large black part, mean male. The cluster which is characterized by the oblique line downward to the right, means the neuter term like cousin. Figure 6.4 is the result of the overlapping clustering method. The result is almost the same as Figure 6.3. But the similarity between aunt and niece and between uncle and nephew is very conspicuous and the feature of cousin is clear comparing Figure 6.3. Moreover, the terms which are characterized by the oblique line downward to the right, mean the collateral family. Figure 6.5 is the result of the rank ordered similarity, and this means that the value of degree of belonging is $\{0,1\}$. This method is to classify roughly, comparing the above simple additive clustering or the overlapping additive clustering, because this method is based on the information of the rank order of the similarity.

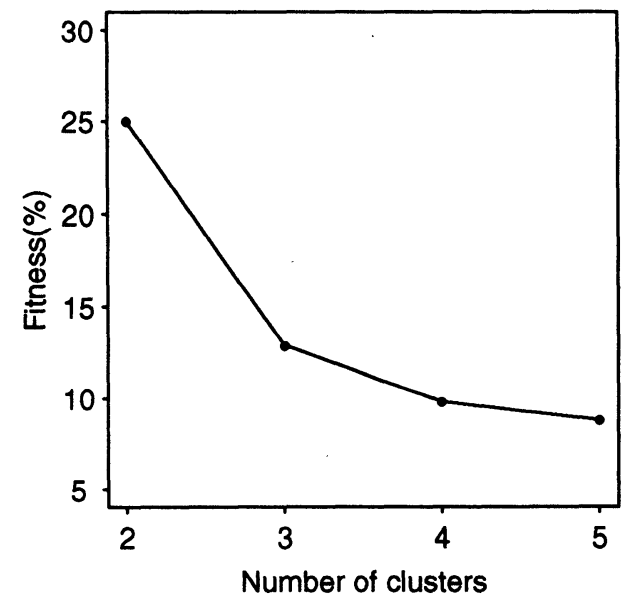

Figure 6.1 Number of clusters and fitness (Simple additive fuzzy clustering)

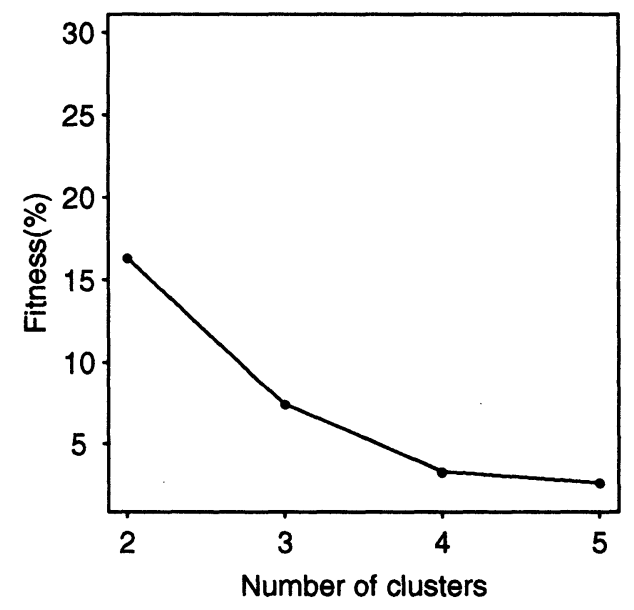

Figure 6.2 Number of clusters and fitness (Overlapping additive fuzzy clustering) 
Then in the case of these data, the hard clustering result is obtained.

Table 6.6 shows the result when we apply the symmetric similarity data (shown in Table 6.2) into the model (5.1). From this, we can find that the values of nondiagonal elements of similarity matrix (shown in Table 6.6) are approximately 0 , that is, the clusters are not similar to each other. Then this shows the validity of the result in Figure 6.3, because we need not consider the similarity between the clusters in these data.

To show the applications of the model (5.1) or (5.3), we use the asymmetric similarity data shown in Table 6.7 (Yoshiharu Sato (1992)). The data show the human relations among 16 children and the value of the data shows the degree of likes and dislikes between the children. The results are shown in Tables 6.8 and 6.9. The number of clusters is determined as 5 based on the value of fitness (shown in Figures 6.6 and 6.7). In Tables 6.8 and 6.9, $C_{1}, C_{2}$, $C_{3}, C_{4}$, and $C_{5}$ represent the five clusters, and the children who belong to a same cluster are good friends with each other. Especially, $C_{1}$ and $C_{2}$ are clusters which consist of only boys, whereas $C_{3}, C_{4}$, and $C_{5}$ are only girls. Moreover, $w_{k l}$ means the relationship between a pair of clusters. For example, the boys who belong to cluster $C_{2}$ have an interest in the girls who belong to cluster $C_{5}$, because the value of $w_{25}$ is large in Tables 6.8 and 6.9. On the other hand, the girls of $C_{5}$ are not interested in boys of $C_{2}$, because the value of $w_{52}$ is small.

Table 6.3 Simple clustering

\begin{tabular}{|c|c|c|c|}
\hline term & $C_{1}$ & $C_{2}$ & $C_{3}$ \\
\hline $\mathrm{a}$ & 0.39 & 0.00 & 0.61 \\
$\mathrm{~b}$ & 0.08 & 0.81 & 0.11 \\
$\mathrm{c}$ & 0.97 & 0.03 & 0.00 \\
$\mathrm{~d}$ & 0.06 & 0.09 & 0.85 \\
$\mathrm{f}$ & 0.01 & 0.85 & 0.14 \\
$\mathrm{gd}$ & 0.15 & 0.06 & 0.79 \\
gf & 0.15 & 0.75 & 0.10 \\
gm & 0.18 & 0.05 & 0.77 \\
gs & 0.11 & 0.80 & 0.09 \\
$\mathrm{~m}$ & 0.05 & 0.08 & 0.86 \\
$\mathrm{ne}$ & 0.35 & 0.65 & 0.00 \\
ni & 0.39 & 0.00 & 0.61 \\
$\mathrm{si}$ & 0.11 & 0.07 & 0.81 \\
so & 0.02 & 0.83 & 0.15 \\
$\mathrm{u}$ & 0.35 & 0.65 & 0.00 \\
\hline \multicolumn{4}{|c}{$\eta_{1}^{2}=0.1287$}
\end{tabular}

Table 6.4 Overlapping clustering

\begin{tabular}{|c|c|c|c|}
\hline term & $C_{1}$ & $C_{2}$ & $C_{3}$ \\
\hline $\mathrm{a}$ & 0.72 & 0.00 & 0.78 \\
$\mathrm{~b}$ & 0.00 & 0.97 & 0.14 \\
$\mathrm{c}$ & 0.47 & 0.11 & 0.12 \\
$\mathrm{~d}$ & 0.02 & 0.12 & 1.00 \\
$\mathrm{f}$ & 0.00 & 1.00 & 0.13 \\
$\mathrm{gd}$ & 0.06 & 0.09 & 1.00 \\
$\mathrm{gf}$ & 0.00 & 0.90 & 0.12 \\
$\mathrm{gm}$ & 0.03 & 0.06 & 0.92 \\
$\mathrm{gs}$ & 0.01 & 1.00 & 0.14 \\
$\mathrm{~m}$ & 0.00 & 0.09 & 1.00 \\
$\mathrm{ne}$ & 0.72 & 0.85 & 0.00 \\
$\mathrm{ni}$ & 0.77 & 0.00 & 0.79 \\
$\mathrm{si}$ & 0.00 & 0.09 & 1.00 \\
$\mathrm{so}$ & 0.00 & 1.00 & 0.18 \\
$\mathrm{u}$ & 0.67 & 0.81 & 0.00 \\
\hline \multicolumn{4}{|c}{$\eta_{1}^{2}=0.0742$}
\end{tabular}


Table 6.5 Ordered clustering

\begin{tabular}{|c|c|c|c|}
\hline term & C1 & C2 & C3 \\
\hline $\mathrm{a}$ & 0.00 & 0.00 & 1.00 \\
$\mathrm{~b}$ & 0.00 & 1.00 & 0.00 \\
$\mathrm{c}$ & 1.00 & 0.00 & 0.00 \\
$\mathrm{~d}$ & 0.00 & 0.00 & 1.00 \\
$\mathrm{f}$ & 0.00 & 1.00 & 0.00 \\
$\mathrm{gd}$ & 0.00 & 0.00 & 1.00 \\
$\mathrm{gf}$ & 0.00 & 1.00 & 0.00 \\
$\mathrm{gm}$ & 0.00 & 0.00 & 1.00 \\
$\mathrm{gs}$ & 0.00 & 1.00 & 0.00 \\
$\mathrm{~m}$ & 0.00 & 0.00 & 1.00 \\
$\mathrm{ne}$ & 0.00 & 1.00 & 0.00 \\
$\mathrm{ni}$ & 0.00 & 0.00 & 1.00 \\
$\mathrm{si}$ & 0.00 & 0.00 & 1.00 \\
$\mathrm{so}$ & 0.00 & 1.00 & 0.00 \\
$\mathrm{u}$ & 0.00 & 1.00 & 0.00 \\
\hline \multicolumn{4}{|c}{$\eta_{2}^{2}=0.0001$}
\end{tabular}

Table 6.6 Simple clustering for asymmetric similarity

\begin{tabular}{|c|c|c|c|}
\hline term & C1 & C2 & C3 \\
\hline a & 0.38 & 0.00 & 0.62 \\
b & 0.07 & 0.82 & 0.10 \\
c & 1.00 & 0.00 & 0.00 \\
d & 0.03 & 0.11 & 0.87 \\
f & 0.00 & 0.87 & 0.13 \\
gd & 0.13 & 0.06 & 0.80 \\
gf & 0.15 & 0.76 & 0.09 \\
gm & 0.16 & 0.06 & 0.78 \\
gs & 0.12 & 0.81 & 0.07 \\
m & 0.01 & 0.11 & 0.88 \\
ne & 0.36 & 0.64 & 0.00 \\
ni & 0.39 & 0.00 & 0.61 \\
si & 0.09 & 0.09 & 0.83 \\
so & 0.01 & 0.85 & 0.14 \\
u & 0.36 & 0.64 & 0.00 \\
\hline \multicolumn{4}{|c}{$\eta_{3}^{2}=0.1321$}
\end{tabular}

Asymmetric matrix

\begin{tabular}{|c|c|c|c|}
\hline cluster & C1 & C2 & C3 \\
\hline C1 & 1.00 & 0.05 & 0.05 \\
C2 & 0.05 & 1.00 & 0.00 \\
C3 & 0.05 & 0.00 & 1.00 \\
\hline
\end{tabular}

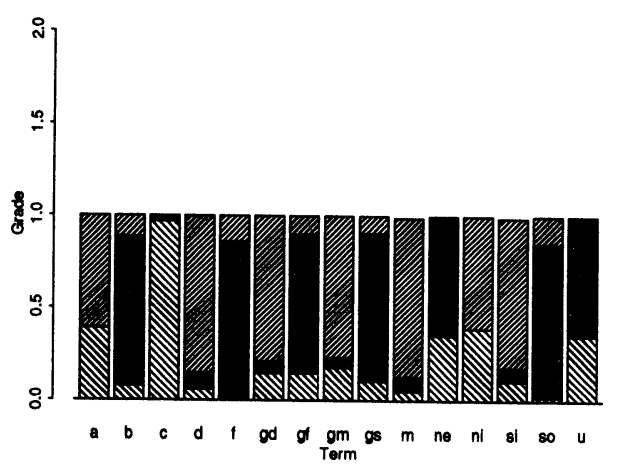

Figure 6.3 Simple clustering of term

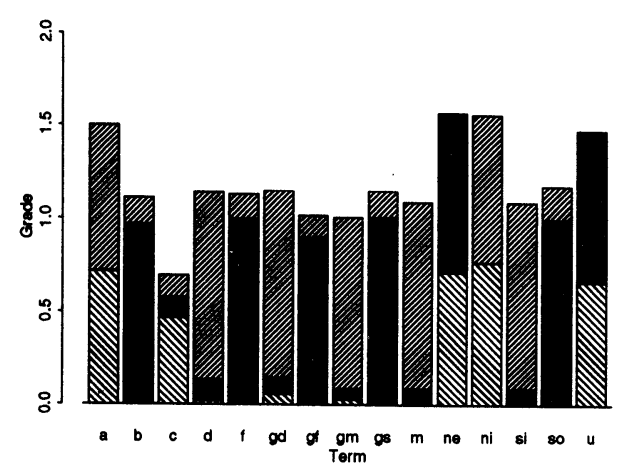

Figure 6.4 Overlapping clustering of term 


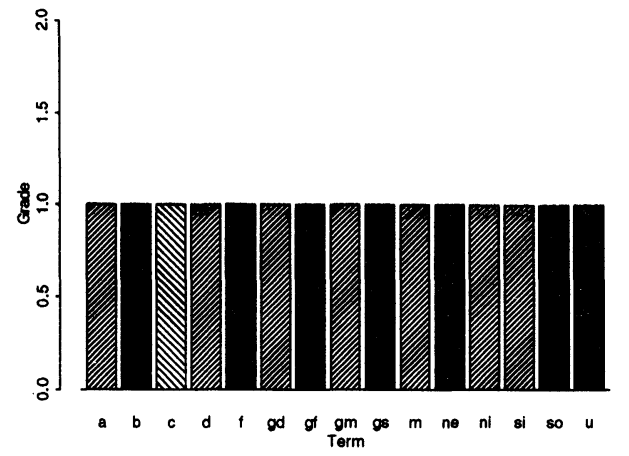

Figure 6.5 Ordered clustering of term

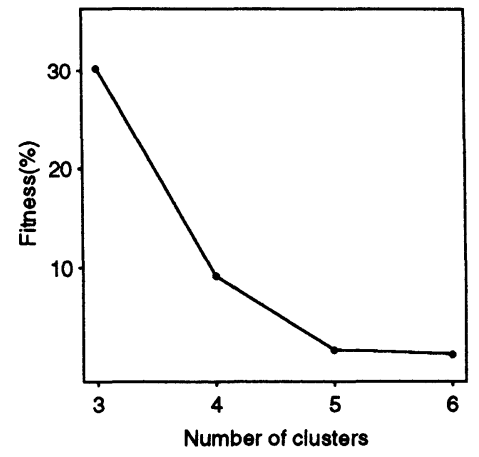

Figure 6.7 Number of clusters and fitness (Overlapping additive fuzzy clustering for asymmetric similarity)

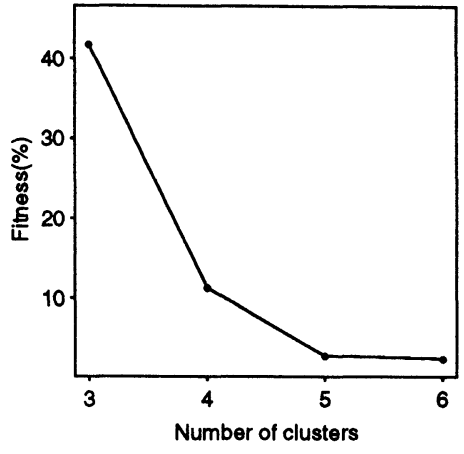

Figure 6.6 Number of clusters and fitness (Simple additive fuzzy clustering for asymmetric similarity) 
Table 6.7 Dissimilarity matrix of children

\begin{tabular}{|c|c|c|c|c|c|c|c|c|c|c|c|c|c|c|c|c|}
\hline child & 1 & 2 & 3 & 4 & 5 & 6 & 7 & 8 & 9 & 10 & 11 & 12 & 13 & 14 & 15 & 16 \\
\hline 1 & 0 & 2 & 3 & 3 & 1 & 1 & 2 & 1 & 3 & 6 & 2 & 3 & 6 & 4 & 6 & 4 \\
\hline 2 & 6 & 0 & 1 & 1 & 6 & 6 & 6 & 6 & 1 & 6 & 6 & 2 & 6 & 2 & 6 & 2 \\
\hline 3 & 6 & 1 & 0 & 2 & 6 & 6 & 6 & 6 & 1 & 6 & 6 & 1 & 6 & 2 & 6 & 2 \\
\hline 4 & 6 & 1 & 2 & 0 & 6 & 6 & 6 & 6 & 1 & 6 & 6 & 2 & 6 & 1 & 6 & 2 \\
\hline 5 & 1 & 3 & 3 & 4 & 0 & 1 & 1 & 2 & 4 & 6 & 3 & 3 & 6 & 4 & 6 & 4 \\
\hline 6 & 1 & 3 & 2 & 4 & 1 & 0 & 2 & 2 & 3 & 6 & 3 & 2 & 6 & 3 & 6 & 3 \\
\hline 7 & 1 & 3 & 3 & 4 & 1 & 1 & 0 & 2 & 4 & 6 & 3 & 3 & 6 & 4 & 6 & 4 \\
\hline 8 & 6 & 1 & 2 & 2 & 6 & 6 & 6 & 0 & 2 & 6 & 1 & 3 & 6 & 3 & 6 & 3 \\
\hline 9 & 6 & 6 & 6 & 6 & 6 & 6 & 6 & 6 & 0 & 6 & 6 & 1 & 6 & 1 & 6 & 1 \\
\hline 10 & 6 & 2 & 3 & 3 & 6 & 6 & 6 & 2 & 3 & 0 & 1 & 4 & 1 & 4 & 1 & 4 \\
\hline 11 & 6 & 1 & 2 & 2 & 6 & 6 & 6 & 1 & 2 & 6 & 0 & 3 & 6 & 3 & 6 & 3 \\
\hline 12 & 6 & 6 & 6 & 6 & 6 & 6 & 6 & 6 & 1 & 6 & 6 & 0 & 6 & 1 & 6 & 1 \\
\hline 13 & 6 & 2 & 3 & 3 & 6 & 6 & 6 & 2 & 3 & 1 & 1 & 4 & 0 & 4 & 1 & 4 \\
\hline 14 & 6 & 6 & 6 & 6 & 6 & 6 & 6 & 6 & 1 & 6 & 6 & 1 & 6 & 0 & 6 & 1 \\
\hline 15 & 6 & 2 & 3 & 3 & 6 & 6 & 6 & 2 & 3 & 1 & 1 & 4 & 1 & 4 & 0 & 4 \\
\hline 16 & 6 & 6 & 6 & 6 & 6 & 6 & 6 & 6 & 1 & 6 & 6 & 1 & 6 & 1 & 6 & 0 \\
\hline
\end{tabular}

Table 6.8 Simple clustering for asymmetric similarity

\begin{tabular}{|c|c|c|c|c|c|}
\hline child & C1 & C2 & C3 & C4 & C5 \\
\hline 1 & 0.94 & 0.00 & 0.04 & 0.02 & 0.00 \\
2 & 0.00 & 0.91 & 0.09 & 0.00 & 0.00 \\
3 & 0.06 & 0.87 & 0.04 & 0.00 & 0.03 \\
4 & 0.00 & 0.88 & 0.00 & 0.08 & 0.04 \\
5 & 0.98 & 0.00 & 0.00 & 0.02 & 0.00 \\
6 & 0.94 & 0.04 & 0.00 & 0.01 & 0.01 \\
7 & 0.95 & 0.00 & 0.00 & 0.05 & 0.00 \\
8 & 0.06 & 0.00 & 0.94 & 0.00 & 0.00 \\
9 & 0.00 & 0.06 & 0.00 & 0.00 & 0.94 \\
10 & 0.00 & 0.00 & 0.00 & 1.00 & 0.00 \\
11 & 0.00 & 0.00 & 0.91 & 0.09 & 0.00 \\
12 & 0.05 & 0.00 & 0.00 & 0.00 & 0.95 \\
13 & 0.00 & 0.00 & 0.00 & 1.00 & 0.00 \\
14 & 0.00 & 0.00 & 0.00 & 0.03 & 0.97 \\
15 & 0.00 & 0.00 & 0.00 & 1.00 & 0.00 \\
16 & 0.02 & 0.00 & 0.00 & 0.03 & 0.95 \\
\hline \multicolumn{5}{|c}{$\eta_{4}^{2}=0.0292$} \\
\hline
\end{tabular}

Asymmetric matrix

\begin{tabular}{|c|c|c|c|c|c|}
\hline cluster & C1 & C2 & C3 & C4 & C5 \\
\hline C1 & 0.92 & 0.47 & 0.66 & 0.00 & 0.42 \\
C2 & 0.01 & 0.98 & 0.02 & 0.01 & 0.77 \\
C3 & 0.00 & 0.76 & 0.98 & 0.00 & 0.56 \\
C4 & 0.00 & 0.56 & 0.77 & 0.88 & 0.37 \\
C5 & 0.01 & 0.00 & 0.00 & 0.01 & 0.92 \\
\hline
\end{tabular}

Table 6.9 Overlapping clustering for asymmetric similarity

\begin{tabular}{|c|c|c|c|c|c|}
\hline child & C1 & C2 & C3 & C4 & C5 \\
\hline 1 & 0.93 & 0.00 & 0.02 & 0.01 & 0.00 \\
2 & 0.00 & 0.93 & 0.04 & 0.00 & 0.00 \\
3 & 0.04 & 0.86 & 0.00 & 0.00 & 0.00 \\
4 & 0.00 & 0.83 & 0.00 & 0.01 & 0.00 \\
5 & 0.89 & 0.00 & 0.00 & 0.00 & 0.00 \\
6 & 0.91 & 0.02 & 0.00 & 0.00 & 0.01 \\
7 & 0.84 & 0.00 & 0.00 & 0.00 & 0.00 \\
8 & 0.04 & 0.00 & 0.90 & 0.00 & 0.00 \\
9 & 0.00 & 0.05 & 0.00 & 0.01 & 0.95 \\
10 & 0.00 & 0.00 & 0.00 & 0.91 & 0.00 \\
11 & 0.00 & 0.00 & 0.88 & 0.06 & 0.00 \\
12 & 0.05 & 0.00 & 0.00 & 0.00 & 0.91 \\
13 & 0.00 & 0.00 & 0.00 & 0.91 & 0.00 \\
14 & 0.00 & 0.00 & 0.00 & 0.00 & 0.89 \\
15 & 0.00 & 0.00 & 0.00 & 0.91 & 0.00 \\
16 & 0.00 & 0.00 & 0.00 & 0.00 & 0.87 \\
\hline \multicolumn{5}{|c}{$\eta_{5}^{2}=0.0183$} \\
\hline
\end{tabular}

Asymmetric matrix

\begin{tabular}{|c|c|c|c|c|c|}
\hline cluster & C1 & C2 & C3 & C4 & C5 \\
\hline C1 & 1.00 & 0.59 & 0.75 & 0.01 & 0.49 \\
C2 & 0.00 & 1.00 & 0.01 & 0.01 & 0.90 \\
C3 & 0.01 & 0.88 & 1.00 & 0.01 & 0.63 \\
C4 & 0.00 & 0.69 & 0.89 & 1.00 & 0.44 \\
C5 & 0.00 & 0.00 & 0.00 & 0.01 & 1.00 \\
\hline
\end{tabular}




\section{Conclusion}

As the method of the fuzzy clustering in which the observations are given by similarity or dissimilarity, we proposed three additive fuzzy clustering models and two extended additive fuzzy clustering models for asymmetric data. These methods are regarded as an extended method of the method proposed by Shepard, R.N. et al., but there is a difference of the concept of overlapping and the consideration of the order of a similarity.

As the method of fuzzy clustering whose object is a similarity data set, RFCM (relational fuzzy clustering method) (Hathaway, R.J., et al. (1989)) has been proposed. This method is to calculate centroids by using a partition matrix $U=\left(u_{i k}\right)$, minimizing a criterion defined in RFCM (extended within dispersion), when a dissimilarity given as data set is defined as an inner product. But in this proposed method, similarity does not have to be an inner product, and this method can treat of various scales, e.g., a ratio scale, an interval scale, or an ordinal scale. The structure of the similarity seems to be extracted clearly by loosening the condition $\sum_{k=1}^{K} u_{i k}=1$. And the reproduction and the robustness of this method are confirmed by simulating. Furthermore, we extend the model to apply to the asymmetric similarity data. Using this extended model, the structure of an asymmetric similarity between objects can be summarized by the asymmetric similarity between clusters.

\section{Acknowledgements}

The authors wish to thank the referees and the editor for their helpful suggestions.

\section{REFERENCE}

Hathaway, R.J., Davenport, J.W. and Bezdek, J.C. (1989) Relational Duals of The c-Means Clustering Algorithms. Pattern Recognition, 22, pp. 205-212.

Kruskal, J.B. (1964) Multidimensional Scaling by Optimizing Goodness of Fit to a Nonmetric Hypothesis. Psychometrika, 29, pp. 1-27.

Kruskal, J.B. (1964) Nonmetric Multidimensional Scaling: A Numerical Method. Psychometrika, 29, pp. 115-129.

Rosenberg, S. and Kim, M.P. (1975) The Method of Sorting as a Data Gathering Procedure in Multivariate Research. Multivariate Behavioral Research, 10, pp. 489-502.

Shepard, R.N. and Arabie, P. (1979) Additive Clustering: Representation of Similarities as Combinations of Discrete Overlapping Properties. Psychological Review, 86, 2, pp. 87-123. Yoshiharu Sato (1992) Multidimensional Scaling in Minkowski Space. Hokkaido Behavioral Science Report, Series $M$, No.20, pp. 69-99.

\section{Appendix}

We should find the degree of belonging $u_{i k}$ and $\alpha$ satisfying the following:

$$
J=\sum_{i \neq j=1}^{n}\left(s_{i j}-\alpha \sum_{k=1}^{K} u_{i k} u_{j k}\right)^{2} \rightarrow \min
$$


If $U_{i j}=\sum_{k=1}^{K} u_{i k} u_{j k}$, then $(\mathrm{A} 1)$ is

$$
J=\sum_{i \neq j=1}^{n}\left(s_{i j}-\alpha U_{i j}\right)^{2} \rightarrow \min
$$

From $\frac{\partial J}{\partial \alpha}=0$

$$
\alpha=\frac{\sum_{i \neq j=1}^{n}\left(s_{i j}-\bar{s}\right) U_{i j}}{\sum_{i \neq j=1}^{n}\left(U_{i j}-\bar{U}\right)^{2}}
$$

where

$$
\begin{gathered}
\bar{U}=\frac{1}{n(n-1)} \sum_{i \neq j=1}^{n} U_{i j}, \\
\bar{s}=\frac{1}{n(n-1)} \sum_{i \neq j=1}^{n} s_{i j} .
\end{gathered}
$$

The descent vector is determined as follows:

$$
\begin{gathered}
\frac{\partial J}{\partial u_{a r}}=-4 \alpha \sum_{i=1}^{n}\left\{s_{i a}-\alpha U_{i a}\right\} u_{i r}, \\
a=1, \cdots, n, \quad r=1, \cdots, K .
\end{gathered}
$$

The following Newton's method is used to find the solutions $U$ and $\alpha$.

(step 1) Fix K, $2 \leq K<n$.

(step 2) Initialize $U^{(0)}=\left(u_{i k}^{(0)}\left(\theta_{i k}\right)\right),(i=1, \cdots, n ; k=1, \cdots, K)$, where $\theta_{i k}$ is generated by uniform pseudorandom numbers in the interval $\left[0, \frac{\pi}{2}\right]$. And set the step number $l=0$.

(step 3) Set $l=l+1$. Calculate $\alpha^{(l)}$ by using $(A 3)$.

(step 4) Calculate the value of $\frac{\partial J}{\partial u_{i k}^{(l-1)}}$ by using $(A 4)$ and $\alpha^{(l)}$ obtained in step 3. Find the optimal solution with respect to the direction of the descent vector by using the one dimensional direct search. That is update $u_{i k}$ by using the next expression,

$$
u_{i k}^{(l)}=u_{i k}^{(l-1)}-\lambda\left(\frac{\partial J}{\partial u_{i k}^{(l-1)}}\right)
$$

where $\lambda>0$ is step size.

(step 5) Calculate the value of $\left\|U^{(l)}-U^{(l-1)}\right\|$ by using $\alpha^{(l)}$ and $u_{i k}^{(l)}$ obtained in step 3 and step 4 , respectively, where $\|\cdot\|$ shows the norm of matrix. 
(step 6) If $\left\|U^{(l)}-U^{(l-1)}\right\|<\varepsilon$, then stop, or else go to step 3. satisfies

In the case of the model for interval scaled similarity, we determine $u_{i k}, \alpha, \beta$ which

$$
J=\sum_{i \neq j=1}^{n}\left(s_{i j}-\alpha \sum_{k=1}^{K} u_{i k} u_{j k}-\beta\right)^{2} \rightarrow \min .
$$

From $\frac{\partial J}{\partial \beta}=0$,

$$
\begin{gathered}
\beta=\bar{s}-\alpha \bar{U} \\
\frac{\partial J}{\partial u_{a r}}=-4 \alpha \sum_{i=1}^{n}\left(s_{i a}-\alpha U_{i a}-\beta\right) u_{i r}
\end{gathered}
$$

In $(A 6)$, we find $(A 3)$ from $\frac{\partial J}{\partial \alpha}=0$.

The algorithm of the model for ordinal scaled similarity is the following: $\frac{\partial \eta_{2}^{2}}{\partial u_{a r}}$ is given with respect to $\eta_{2}^{2}$.

$$
\begin{aligned}
\eta_{2}^{2} & =\frac{A}{B} \\
A & =\sum_{i \neq j=1}^{n}\left(w_{i j}-\tilde{w}_{i j}\right)^{2} \\
B & =\sum_{i \neq j=1}^{n}\left(w_{i j}-\bar{w}\right)^{2} \\
\frac{\partial \eta_{2}^{2}}{\partial u_{a r}} & =\frac{1}{B} \frac{\partial A}{\partial u_{a r}}-\frac{A}{B^{2}} \frac{\partial B}{\partial u_{a r}} \\
\frac{\partial A}{\partial u_{a r}} & =2 \sum_{i \neq j}\left(w_{i j}-\tilde{w}_{i j}\right)\left(\frac{\partial w_{i j}}{\partial u_{a r}}-\frac{\partial \tilde{w}_{i j}}{\partial u_{a r}}\right) \\
& =2 \sum_{i \neq j}\left(w_{i j}-\tilde{w}_{i j}\right) \frac{\partial w_{i j}}{\partial u_{a r}} \\
\frac{\partial B}{\partial u_{a r}} & =2 \sum_{i \neq j}\left(w_{i j}-\bar{w}\right)\left(\frac{\partial w_{i j}}{\partial u_{a r}}-\frac{\partial \bar{w}}{\partial u_{a r}}\right) \\
& =2 \sum_{i \neq j}\left(w_{i j}-\bar{w}\right) \frac{\partial w_{i j}}{\partial u_{a r}}
\end{aligned}
$$

(step 1) Fix K, $2 \leq K<n$.

(step 2) Initialize $U^{(0)}=\left(u_{i k}^{(0)}\left(\theta_{i k}\right)\right),(i=1, \cdots, n ; k=1, \cdots, K)$, where $\theta_{i k}$ is generated by uniform pseudorandom numbers in the interval $\left[0, \frac{\pi}{2}\right]$. And set the step number $l=0$. 
(step 3) Set $l=l+1$. Calculate $W=U^{(l-1)}\left(U^{(l-1)}\right)^{t}$, where $t$ is a transposed representation.

(step 4) Find $\tilde{W}$ based on the monotone regression principle.

(step 5) Calculate the value of $\frac{\partial \eta_{2}^{2}}{\partial u_{i k}^{(l-1)}}$, find the optimal solution

$$
u_{i k}^{(l)}=u_{i k}^{(l-1)}-\lambda\left(\frac{\partial \eta_{2}^{2}}{\partial u_{i k}^{(l-1)}}\right)
$$

by using the one dimensional direct research, where $\lambda$ is step size.

(step 6) If $\left\|U^{(l)}-U^{(l-1)}\right\|<\varepsilon$, then stop, or else go to step 3 .

(Received October 1993; Revised October 1994) 